\title{
A Theorem on Bimeromorphic Maps of Kähler Manifolds and Its Applications
}

By

\author{
Akira FujıKI
}

\section{Introduction}

For a compact complex manifold $Z$ we denote by $P(Z)$ (resp. $S P(Z)$ ) the convex cone in $H^{2}(Z, \mathbb{R})$ consisting of classes which are positive (resp. semipositive) in the sense of Kodaira. Thus we have $P(Z) \subseteq S P(Z) \cong H^{1,1}(Z) \cong H^{2}(Z, \mathbb{R})$. In particular $Z$ is Kähler if and only if $P(Z) \neq \varnothing$, and in this case $\operatorname{SP}(Z)$ is the closure of $P(Z)$ in $H^{2}(Z, \boldsymbol{R})$.

Now let $f: X \rightarrow Y$ be a bimeromorphic map of compact complex manifolds. Suppose that $f$ induces an isomorphism of complements of analytic subsets of codimension $\geqq 2$. Then as a main theorem of this note we shall show that either $f$ is biholomorphic or $f_{*}(P(X)) \cap S P(X)=\varnothing$ in $H^{2}(Y, \mathbb{R})$, where $f_{*}$ : $H^{2}(X, \mathbb{R}) \rightarrow H^{2}(Y, \boldsymbol{R})$ is the homomorphism induced by $f$. (See Theorem 3.2 for a little more general statement.) In particular if $X$ is projective with an ample divisor $D$ and if the linear system $\left|f_{*} D\right|$ is base point free on $Y$, then $f$ must be biholomorphic, the fact which can be verified directly using the natural isomorphism $\Gamma\left(X, \mathcal{O}_{X}(D)\right) \cong \Gamma\left(Y, \mathcal{O}_{Y}\left(f_{*} D\right)\right)$. However, in $[4,(1.13)]$ we have given another proof for this in a certain special case, which in fact is applicable also to the general case in view of Lemma 3.1 below and of the transformation formula (8) in Lemma 2.4, well-known for divisors. The advantage of the latter proof lies in the fact that it can further be generalized to give the main theorem as above. For this purpose, since a Kähler class, or more generally, a semipositive class cannot in general be represented by divisors, as substitutes we consider positive currents of type $(1,1)$ in the sense of Lelong [10]. They include as special cases semipositive forms on the one hand, and effective

Received November 22, 1980.

* Research Institute for Mathematical Sciences, Kyoto University. Current address: Yoshida College, Kyoto University, Kyoto 606 Japan. Supported by the Sakkokai foundation 
divisors on the other hand. Moreover they are transformed just as divisors under bimeromorphic maps; indeed the transformation formula (8) mentioned above is verified for them. Once (8) is established the proof of the theorem is essentially the same as in the case of divisors, though in the actual proof we do not separate the case of divisors.

In Section 2 some lemmas on positive currents including Lemma 2.4 will be shown and then in Section 3 Theorem 3.2 and some of its corollaries will be given. Section 1 is devoted to some preliminary study on the functorial behavior of line bundles and their chern classes under bimeromorphic maps. On the other hand, in Section 4 as the main application of our theorem we shall give a kählerian analogue of a theorem of Matsusaka-Mumford [11] concerning the uniqueness of the limit in smooth deformation of polarized manifolds (Theorem 4.3). This result will play a fundamental role in our construction of the coarse moduli space for polarized family of compact Kähler manifolds in [5]. As a more specific application we also prove that every bimeromorphic automorphism of a compact Kähler manifold $X$ with $h^{1,1}(X)=1$ and $c_{1}(X)=0$ is necessarily biholomorphic (Proposition 3.6).

In this paper complex manifolds are assumed to be paracompact and connected. A complex space $E$ of pure codimension 1 in a complex manifold $X$ is called an effective divisor, or simply a divisor when no confusion may arise. In this case we denote by $[E]$ the line bundle defined by $E$. Except for the case of divisors complex spaces are in general assumed to be reduced.

\section{§1. Bimeromorphic Maps, Line Bundles and Chern Classes}

Let $f: X \rightarrow Y$ be a morphism of complex spaces. Then $f$ is called bimeromorphic if $f$ is proper and there exists a dense Zariski open subset $U$ (resp. $V$ ) of $X$ (resp. $Y$ ) such that $f$ induces an isomorphism of $U$ and $V$. In this case there exist in fact unique maximal such $U$ and $V$, which will again be denoted by the same letters $U$ and $V$ respectively. Then we call $E=X-U$ the exceptional set of $f$. Let $F=Y-V(=f(E))$. Then $\operatorname{codim} F \geqq 2$ when $Y$ is normal and $E$ is of pure codimension 1 when $Y$ is nonsingular (cf. [6]).

Let $X$ and $Y$ be complex spaces as above. Then a meromorphic map $f: X \rightarrow Y$ is an analytic subspace $\Gamma$ of $X \times Y$ called the graph of $f$, such that the natural projection $\pi_{1}: \Gamma \rightarrow X$ is bimeromorphic. We call $f$ bimeromorphic, if the other projection $\pi_{2}: \Gamma \rightarrow Y$ also is bimeromorphic. In this case $f^{-1}: Y \rightarrow X$ 
is by definition the bimeromorphic map with the graph ${ }^{t} \Gamma=\{(y, x) \in Y \times X$; $(x, y) \in \Gamma\} \subseteq Y \times X$.

Let $X$ be a complex manifold and $\left(\mathscr{E}_{X}^{\cdot}, d\right)\left(\right.$ resp. $\left.\left({ }^{\circ}{ }_{X}^{\circ}, d\right)\right)$ be the complex of sheaves of germs of real valued $C^{\infty}$ forms (resp. currents) on $X$. Then we have the usual de Rham isomorphisms $H^{i}(X, \mathbb{R}) \cong H^{i} \Gamma\left(X, \mathscr{E}_{X}^{*}\right) \cong H^{i} \Gamma\left(X,{ }^{\prime} \mathscr{D}_{X}^{\circ}\right)$. Let $\mathscr{E}_{X}^{p, p} \subseteq \mathscr{E}_{X}^{2 p}$ (resp. ' $\left.\mathscr{D}_{X}^{p}, \subseteq^{\prime} \mathscr{D}_{X}^{2 p}\right)$ be the subsheaf of germs of type $(p, p), p>0$. Then we put $\mathscr{Z}_{X}^{1}, 1=\operatorname{Ker}\left(d: \mathscr{E}_{X}^{1}, 1 \rightarrow \mathscr{E}_{X}^{3}\right)\left(\operatorname{resp} .^{\prime} \mathscr{Z}_{X}^{1}, 1=\operatorname{Ker}\left(d:{ }^{\prime} \mathscr{D}_{X}^{1}, 1 \rightarrow^{\prime} \mathscr{D}_{X}^{3}\right)\right)$ and $\hat{H}^{1,1}(X)=\Gamma\left(X, \mathscr{Z}_{X}^{1}, 1\right) / d d^{c} \Gamma\left(X, \mathscr{E}_{X}^{0}\right)\left(\right.$ resp. ' $\left.\hat{H}^{1,1}(X)=\Gamma\left(X, \mathscr{Z}_{X}^{1}, 1\right) / d d^{c} \Gamma\left(X, \mathscr{D}_{X}^{0}\right)\right)$ where $d^{c}=\sqrt{-1}(\bar{\partial}-\partial), \partial$ (resp. $\left.\bar{o}\right)$ being the $(1,0)$ (resp. $(0,1)$ ) component of d. Let $\mathscr{P}_{X}$ be the sheaf of germs of pluriharmonic functions on $X$. Then we have the following commutative diagram of exact sequences

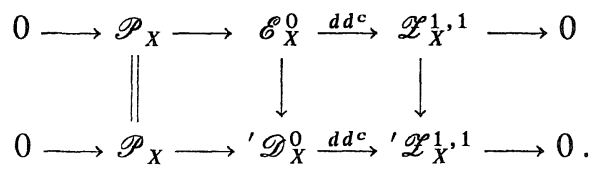

From this we get the natural isomorphisms

$$
\hat{H}^{1,1}(X) \cong H^{1}\left(X, \mathscr{P}_{X}\right) \cong \hat{H}^{1,1}(X) .
$$

Then we denote by $H^{1,1}(X)$ the isomorphism class of these vector spaces. In what follows, however, we often identify $H^{1,1}(X)$ with any of these vector spaces. The natural map ' $\hat{H}^{1,1}(X) \rightarrow H^{2}(X, \mathbb{R})$ induced by the inclusion ' $\mathscr{D}{ }_{X}^{1}, 1$ $\subseteq^{\prime} \mathscr{D}_{X}^{2}$ defines a linear map

$$
\lambda: H^{1,1}(X) \longrightarrow H^{2}(X, \mathbb{R}) .
$$

We consider the following commutative diagram of short exact sequences

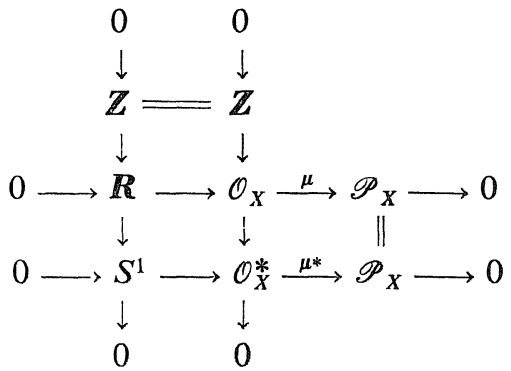

where $S^{1}$ is the circle, $\mu$ and $\mu^{*}$ are defined respectively by $\mu(f)=-$ (imaginary part of $f$ ) and $\mu^{*}(f)=(1 / 2 \pi) \log |f|$, and the middle vertical line is the usual exponential exact sequence. From this we have the following commutative 
diagram of cohomology exact sequences

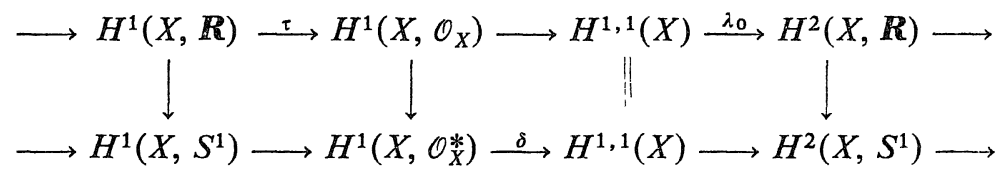

where we have identified $H^{1}\left(X, \mathscr{P}_{X}\right)$ with $H^{1,1}(X)$. Then for each holomorphic line bundle $L \in H^{1}\left(X, \mathcal{O}_{X}^{*}\right)$ we define its refined chern class $\hat{c}(L) \in H^{1,1}(X)$ by $\hat{c}(L)=\delta(L)$ (cf. [14]). On the other hand, $\lambda_{0}$ is easily identified with the $\lambda$ above. In particular $\lambda$ is injective if and only if $\tau$ is surjective. The latter is the case if, e.g., $X$ is compact and bimeromorphic to a compact Kähler manifold (cf. [3]).

Let $f: X \rightarrow Y$ be a proper morphism of complex manifolds. Let $\tilde{f}^{*}$ : $\Gamma\left(Y, \mathscr{E}_{Y}^{\circ}\right) \rightarrow \Gamma\left(X, \mathscr{E}_{X}^{*}\right)$ be the pull-back of forms and $\tilde{f}_{*}: \Gamma\left(X,{ }^{\prime} \mathscr{D}_{X}^{*}\right) \rightarrow \Gamma\left(Y,{ }^{\prime} \mathscr{D} \dot{Y}_{Y}^{\circ}\right)$ the direct image of currents. Since they commute with $d$ and $d^{c}$ and are compatible with types, they induce the natural homomorphisms $f^{*}: H^{i}(Y, \boldsymbol{R}) \rightarrow$ $H^{i}(X, \boldsymbol{R}), f^{*}: H^{1,1}(Y) \rightarrow H^{1,1}(X)$ and $f_{*}: H^{i}(X, \boldsymbol{R}) \rightarrow H^{i}(Y, \boldsymbol{R}), f_{*}: H^{1,1}(X) \rightarrow$ $H^{1,1}(Y)$ respectively. Suppose now that $f$ is bimeromorphic. Then $\tilde{f}_{*} \tilde{f}^{*}$ is the identity on $\Gamma\left(Y, \mathscr{E}_{Y}^{*}\right)\left(\subseteq \Gamma\left(Y,{ }^{\prime} \mathscr{D}_{Y}^{\cdot}\right)\right)$ and hence so is $f_{*} f^{*}$. In particular $H^{i}(Y, \boldsymbol{R})$ (resp. $\left.H^{1,1}(Y)\right)$ is naturally a direct summand of $H^{i}(X, \boldsymbol{R})$ (resp. $\left.H^{1,1}(X)\right)$.

On the other hand, (still under the assumption that $f$ is bimeromorphic) we can also define

$$
f_{*}: H^{1}\left(X, \mathcal{O}_{X}^{*}\right) \longrightarrow H^{1}\left(Y, \mathcal{O}_{Y}^{*}\right)
$$

for the spaces of line bundles as well as the usual pull-back homomorphism $f^{*}: H^{1}\left(Y, \mathcal{O}_{Y}^{*}\right) \rightarrow H^{1}\left(X, \mathcal{O}_{X}^{*}\right)$. Indeed, let $E$ be the exceptional set of $f, F=f(E)$, $U=X-E$, and $V=Y-F$. First note that $\mathscr{H}_{F}^{i}\left(\mathcal{O}_{Y}^{*}\right)=0, i=0,1$, so that $H_{F}^{1}\left(Y, \mathcal{O}_{Y}^{*}\right)$ $=0, H_{F}^{2}\left(Y, \mathcal{O}_{Y}^{*}\right) \cong H^{0}\left(Y, \mathscr{H}_{F}^{2} \mathcal{O}_{Y}^{*}\right) \cong H^{0}\left(Y, R^{1} j_{*} \mathcal{O}_{V}^{*}\right)$, where $j: V \rightarrow Y$ is the inclusion. So we have the following commutative diagram

$$
\begin{array}{ll}
H^{1}\left(X, \mathcal{O}_{X}^{*}\right) \stackrel{r_{U}}{\longrightarrow} H^{1}\left(U, \mathcal{O}_{U}^{*}\right) \\
\downarrow_{\downarrow} \stackrel{\imath}{f_{*}} & (\downarrow \\
H^{1}\left(Y, \mathcal{O}_{Y}^{*}\right) \stackrel{r_{V}}{\longrightarrow} H^{1}\left(V, \mathcal{O}_{V}^{*}\right) \stackrel{\delta}{\longrightarrow} H^{0}\left(Y, R^{1} j_{*} \mathcal{O}_{V}^{*}\right)
\end{array}
$$

where $r_{U}, r_{V}$ are restriction maps with $r_{V}$ injective, and $\delta$ is the coboundary homomorphism $H^{1}\left(V, \mathcal{O}_{V}^{*}\right) \rightarrow H_{F}^{2}\left(Y, \mathcal{O}_{Y}^{*}\right)$ composed with the above isomorphism. We shall show that $\delta \eta r_{U}(L)=0$ for every $L \in H^{1}\left(X, \mathcal{O}_{X}^{*}\right)$. Let $\mathscr{L}=\mathcal{O}_{X}(L)$. 
Then $f_{*} \mathscr{L}$ is a coherent analytic sheaf on $Y$ representing the class $\eta r_{U}(L)$ on $V$. Hence it is enough to show that for every open subset $W$ of $Y$ which is isomorphic to a polydisc, $f_{*} \mathscr{L}$ is isomorphic to $\mathcal{O}_{Y}$ on $W-W \cap Y$. In fact take any nonzero section $s \in \Gamma\left(W, f_{*} \mathscr{L}\right)$. Let $A$ be the union of irreducible components of codimension 1 of the zero of $s$ and $[A]$ the line bundle defined by $A$. Then $f_{*} \mathscr{L} \cong \mathcal{O}_{Y}([A])$ on $W-W \cap Y$, while $\mathcal{O}_{Y}([A])=\mathcal{O}_{Y}$ on $W$ since $W$ is isomorphic to a polydisc. This proves our assertion. It then follows from the above diagram that there exists a unique $L^{\prime} \in H^{1}\left(Y, \mathcal{O}_{Y}^{*}\right)$ such that $r_{V}\left(L^{\prime}\right)=\eta r_{U}(L)$. Then $\int_{*} L$ $=L^{\prime}$ by definition.

In this case we also have $f_{*} f^{*}=$ identity on $H^{1}\left(Y, \mathcal{O}_{Y}^{*}\right)$ (projection formula) and hence $H^{1}\left(Y, \mathcal{O}_{i}^{*}\right)$ is naturally a direct summand of $H^{1}\left(X, \mathcal{O}_{Y}^{*}\right)$.

Now we shall study the kernel of $f_{*}$. For this purpose let $E_{v}, v=1,2, \ldots$, be the irreducible components of $E$ and $\left[E_{v}\right]$ the line bundles on $X$ defined by $E_{v}$. Further we denote by $e_{v}$ (resp. $\hat{e}_{v}$ ) the real first chern classes $c_{1}\left(\left[E_{v}\right]\right)$ $\in H^{2}(X, \boldsymbol{R})$ (resp. refined chern classes $\hat{c}\left(\left[E_{v}\right]\right) \in H^{1,1}(X)$ ) of $\left[E_{v}\right]$. Then we have following:

Proposition 1.1. Let $f: X \rightarrow Y$ be a bimeromorphic map of complex manifolds as above. Then in the notation above we have the following commutative diagram of (split) exact sequences

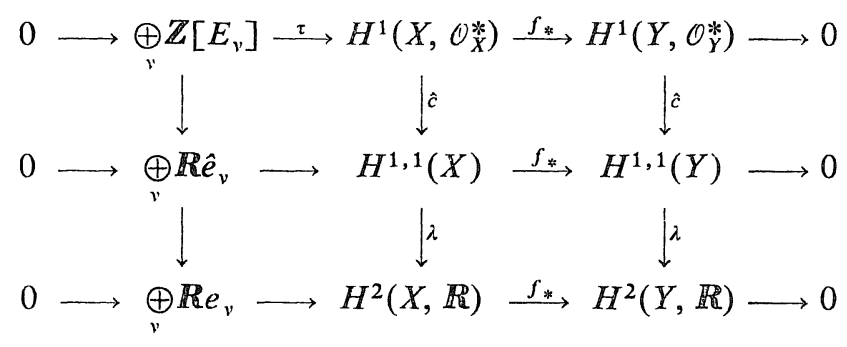

where $\tau\left(\oplus_{v} r_{v}\left[E_{v}\right]\right)=\prod_{v}\left[E_{v}\right]^{\otimes r_{v}}$ and the other maps are either defined above or canonically defined.

Proof. Commutativity. The commutativity of the squares on the left follows by the definition of the maps. (Actually we define the vertical maps on the left by the commutativity of the squares.) The commutativity of the bottom square on the right follows from the definition of $\lambda$ and $f_{*}$. Thus it remains to show that $\hat{c} f_{*}=f_{*} \hat{c}$. First, in view of $\mathscr{H}_{F}^{i}\left(\mathscr{P}_{Y}\right)=0, i=0,1$, using $H^{1,1}(Z) \cong$ $H^{1}\left(Z, \mathscr{P}_{Z}\right), Z=X, Y, U, V$, we get the following diagram of exact sequences 


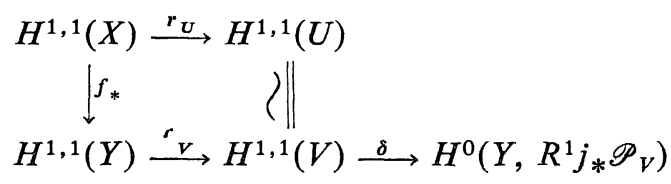

with $r_{V}$ injective in the same way as for (4). Moreover the commutativity of the diagram is obvious. Then considering the natural map from (4) to (5) induced by $\mu^{*}: \mathcal{O}_{\mathbf{Z}}^{*} \rightarrow \mathscr{P}_{Z}$ in (2), we see immediately the desired commutativity.

The exactness of the third sequence. We consider the following commutative diagram of exact sequences of local cohomology

(6)

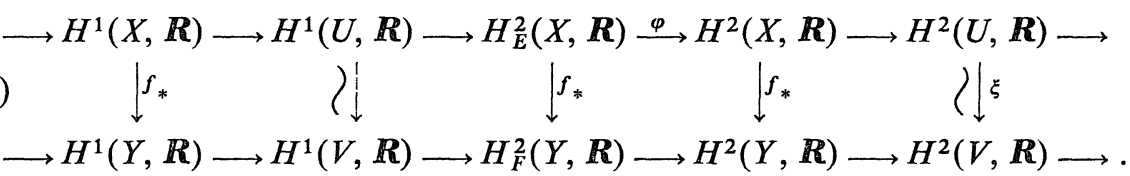

Since $F$ is of codimension $\geqq 2$ in $Y$, we get that $H_{F}^{2}(Y, \boldsymbol{R})=0$. From this and the above sequence it follows immediately that $H_{E}^{2}(X, \boldsymbol{R}) \cong \operatorname{Ker} f_{*}, f_{*}: H^{2}(X, \boldsymbol{R})$ $\rightarrow H^{2}(Y, \boldsymbol{R})$, by $\varphi$. On the other hand, we have the natural isomorphisms $H^{2}(X, \boldsymbol{R}) \cong H^{0}\left(X, \mathscr{H}_{E}^{2}(\boldsymbol{R})\right) \cong \oplus_{v} H^{0}\left(X, \mathscr{H}_{E_{v}}^{2}(\boldsymbol{R})\right) \cong \oplus_{v} H^{0}\left(E_{v}, \boldsymbol{R}\right)$ and the induced isomorphism $\lambda: \oplus_{v} H^{0}\left(E_{v}, \boldsymbol{R}\right) \cong \operatorname{Ker} f_{*}$ is given by $\left(r_{v}\right) \rightarrow \sum_{v} r_{v} e_{v}$, where we identified each $H^{\circ}\left(E_{v}, R^{R}\right)$ naturally with $\boldsymbol{R}$. This shows that the third sequence is exact.

Exactness of the first sequence. By the commutativity of the diagram $\tau$ is injective, and by the definition of $f_{*}$, it is clear that $f_{*} \tau=0$. So it suffices to show that $\operatorname{Ker} f_{*} \subseteq \operatorname{Im} \tau$. Let $L \in \operatorname{Ker} f_{*}$. By commutativity there exists a sequence $\left(r_{v}\right)$ of integers such that $\tilde{c}_{1}\left(L^{\prime}\right)=0$ with $L^{\prime}=\Pi_{v}\left[E_{v}\right]^{r_{v}} \otimes L$, where $\tilde{c}_{1}$ denotes the integral chern class of a line bundle. Consider the following commutative diagram of exponential long exact sequence

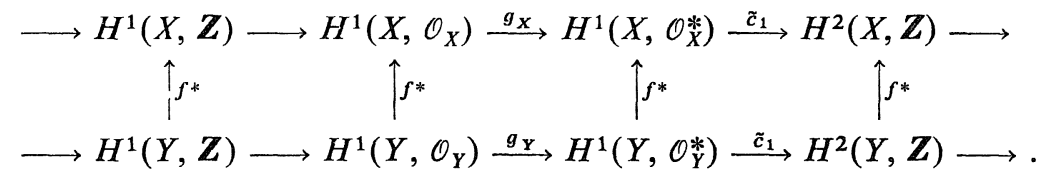

Here the first $f^{*}$ is isomorphic as follows from (6) with $\boldsymbol{R}$ replaced by $\boldsymbol{Z}$, and the second $f^{*}$ is isomorphic since $R^{i} f_{*} \mathcal{O}_{X}=0, i \geqq 1$, by Hironaka [7]. Now $\tilde{c}_{1}\left(L^{\prime}\right)=0$ implies that $L^{\prime}=g_{X}(\beta)$ for some $\beta \in H^{1}\left(X, \mathcal{O}_{X}\right)$. Then $g_{Y} f^{*-1}(\beta)$ $=f_{*} f^{*} g_{Y} f^{*-1}(\beta)=f_{*} g_{X}(\beta)=0$. From these it follows easily that $\beta$ is in the image of $H^{1}(X, Z)$ in the above diagram so that $L^{\prime}$ is trivial. Hence $L \in \operatorname{Im} \tau$. 
Finally the exactness of the second sequence can be proved just as above using the following commutative diagram of exact sequences

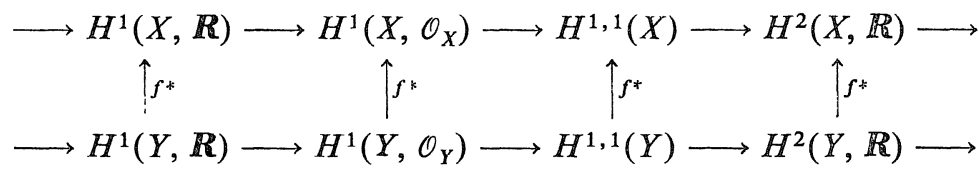

(cf. the top line of (3)) instead of (7), together with $H^{1,1}(Z) \cong H^{1}\left(Z, \mathscr{P}_{Z}\right), Z=X, Y$.

Q.E.D.

A complex manifold $X$ is called relatively minimal if any bimeromorphic morphism $f: X \rightarrow Y$ of $X$ onto a complex manifold $Y$ is necessarily isomorphic.

Corollary 1.2. Let $X$ be a complex manifold with $b_{2}(X)<\infty$, where $b_{2}$ is the second Betti number. Then in the bimeromorphic equivalence class of $X$ there is at least one which is relatively minimal (cf. [9, VIII §3]).

Now in general let $f: X \rightarrow Y$ be a bimeromorphic map of complex manifolds. We shall generalize the definition of $f^{*}$ and $f_{*}$ defined above for morphisms as follows. Take any complex manifold $Z$ with bimeromorphic morphisms $h_{1}: Z \rightarrow X$ and $h_{2}: Z \rightarrow Y$ such that $f=h_{2} h_{1}^{-1}$, which is always possible by resolving the graph of $f$. Then we define $f_{*}: H^{2}(X, \mathbb{R}) \rightarrow H^{2}(Y, \mathbb{R})$ (resp. $\left.H^{1,1}(X) \rightarrow H^{1,1}(Y), H^{1}\left(X, \mathcal{O}_{X}^{*}\right) \rightarrow H^{1}\left(Y, \mathcal{O}_{Y}^{*}\right)\right)$ by $f_{*}=h_{2 *} h_{1}^{*}$, and define $f^{*}$ similarly by $f^{*}=h_{1 *} h_{2}^{*}$. By a standard way we check easily that the definition is in fact independent of the choice of $Z$ as above.

\section{§2. Bimeromorphic Maps and Positive Curremts}

Let $X$ be a complex manifold. Let $\sigma$ be a real closed $(1,1)$ current on $X$, i.e., $\sigma \in \Gamma\left(X, \mathscr{Z}_{X}^{1}, 1\right)$. Then for every point $x \in X$ there exist a neighborhood $U$ of $x$ and a current $\varphi$ of degree zero on $U$ such that $\left.\sigma\right|_{U}=d d^{c} \varphi$. Then according to Lelong [10] we call $\sigma$ positive if $\varphi$ is a plurisubharmonic function for every $x \in X$ and $U$ as above (cf. [10, IV. 4a1)]). The definition is independent of the choice of $\varphi$ because $\varphi$ is unique up to addition of plurisubharmonic functions on $U$. (We refer to [10] for the definition of plurisubharmonic functions and its fundamental properties.) We denote by $\widetilde{P}(X) \subseteq \Gamma\left(X,{ }^{\prime} \mathscr{Z}_{X}^{1}, 1\right)$ the set of positive currents on $X$.

Let $\varphi$ be a plurisubharmonic function defined on an open subset $U$ of $X$. Then we put $S(\varphi)=\{u \in U ; \varphi$ is not locally bounded below at $u\}$, and 
$S_{\infty}(\varphi)=\{u \in U ; \varphi(u)=-\infty\}$. Then we define $S(\sigma)$ for $\sigma \in \widetilde{P}(X)$ by $S(\sigma) \cap U=S(\varphi)$ and $S_{\infty}(\sigma) \cap U=S_{\infty}(\varphi)$ for any open subset $U$ and for any plurisubharmonic function $\varphi$ on $U$ such that $\left.\sigma\right|_{U}=d d^{c} \varphi$. By the same reason as above this is well-defined. By definition $S(\sigma)$ is a closed subset of $X$.

Let $f: X \rightarrow Y$ be a proper morphism of complex manifolds. Let $\sigma$ be a real closed $(1,1)$-current on $X$ and $f_{* \sigma} \sigma$ its direct image. Then if $\sigma$ is positive, $f_{*} \sigma$ is again positive $([10$, p. 70$])$. On the other hand, for any $\tau \in \widetilde{P}(Y)$ with $f(X) \nsubseteq S_{\infty}(\tau)$ we define $f^{*} \tau \in \tilde{P}(X)$ as follows. Take an open covering $U=\left\{U_{i}\right\}$ of $Y$ such that on each $U_{i}$ there is a plurisubharmonic function $\varphi_{i}$ with $\left.\sigma\right|_{U_{i}}$ $=d d^{c} \varphi_{i}$. Then for each $i, \tilde{\varphi}_{i}=\varphi_{i} f$ is a plurisubharmonic function on $\tilde{U}_{i}=$ $f^{-1}\left(U_{i}\right)$ [10, II. 1. Prop. 5]. Further since $\tilde{\varphi}_{i}-\tilde{\varphi}_{j}=\left(\varphi_{i}-\varphi_{j}\right) f$ is pluriharmonic on $U_{i} \cap U_{j},\left\{d d^{c} \tilde{\varphi}_{i}\right\}$ gives an element of $\widetilde{P}(X)$, which is by definition $f^{*} \tau$. The definition is easily seen to be independent of the particular choice of $U$ and $\left\{\varphi_{i}\right\}$ as above and makes sense even if $f$ is not proper. It is clear that $S\left(f^{*} \tau\right)=$ $f^{-1}(S(\tau))$, and $f_{*} f^{*} \tau=\tau$.

We call an element of $H^{1,1}(X)$ (resp. $H^{2}(X, \boldsymbol{R})$ ) current-positive if it is represented by a positive current. We denote by $P_{0}(X)$ (resp. $P(X)$ ) the set of current-positive classes which forms a convex cone in $H^{1,1}(X)\left(\operatorname{resp} . H^{2}(X, \boldsymbol{R})\right)$. If $\alpha \in P_{0}(X)$ (resp. $P(X)$ ), then we set $S(\alpha)=\cap_{\sigma} S(\sigma)$ where $\sigma$ is taken over all the positive currents representing $\alpha$, and call $S(\alpha)$ the set of base points of $\alpha$.

Any effective divisor $A=\sum_{i} n_{i} A_{i}$, where $n_{i}$ are positive integers, $A_{i}$ are irreducible subspaces of codimension 1 in $X$ and the summation is locally finite, defines a positive current $\langle A\rangle=\sum_{i} n_{i}\left\langle A_{i}\right\rangle$ where $\left\langle A_{i}\right\rangle$ is the integration current on $A_{i}$. A (refined) chern class of a line bundle is thus current-positive if it has a nonzero section.

Lemma 2.1. Suppose that $X$ is compact. Then any nonzero positive current $\sigma$ on $X$ defines a nonzero class $[\sigma]$ in $H^{1,1}(X)$. In particular $P_{0}(X)$ contains no straight line.

Proof. Let $\sigma$ be a positive current. If $[\sigma]=0$, then $\sigma=d d^{c} g$ for some $g \in \Gamma\left(X, \mathscr{D}_{X}^{0}\right)$. Since $\sigma$ is positive, $g$ is plurisubharmonic. Hence $g$ is constant since $X$ is compact. Thus $\sigma=0$. If $P_{0}(X)$ contains a straight line, there is a nonzero element $\beta \in H^{1,1}(X)$ such that both $\beta$ and $-\beta$ are current positive. Let $\sigma$ and $\sigma^{\prime}$ be positive currents representing $\beta$ and $-\beta$ respectively. Then $\sigma+\sigma^{\prime} \in \widetilde{P}(X)$ represents zero, so that $\sigma+\sigma^{\prime}=0$ by what we have proved above. The latter is possible only when $\sigma=\sigma^{\prime}=0$. This is a contradiction. Q.E.D. 
Lemma 2.2. Let $f: X \rightarrow Y$ be a morphism of complex manifolds and $\beta \in H^{1,1}(Y)$. Suppose that $\beta$ is current-positive and $\tau$ is a positive current representing $\beta$. Then if $f(X) \nsubseteq S_{u}(\tau), f^{*} \beta \in H^{1,1}(X)$ is represented by $f^{*} \tau$. In particular $f^{*} \beta$ also is current-positive with $S\left(f^{*} \beta\right) \subseteq f^{-1}(S(\beta))$.

Proof. By (1) there exist $\tau^{\prime} \in \Gamma\left(Y, \mathscr{Z}_{Y}^{1}, 1\right)$ and $g \in \Gamma\left(Y, \mathscr{D}_{Y}^{0}\right)$ such that $\tau=\tau^{\prime}+d d^{c} g$. By the definition of $f^{*}, f^{*} \rho$ is represented by $f^{*} \tau^{\prime} \in \Gamma\left(X, \mathscr{Z}_{X}^{1}, 1\right)$. First we note that $g$ is a locally summable function. In fact, for any point $y \in Y$ we may writc $\tau^{\prime}=d d^{c} t$ (resp. $\tau=d d^{c} \varphi$ ) for some $C^{\infty}$ (resp. plurisubharmonic) function $t$ (resp. $\varphi$ ) defined in a neighborhood $W$ of $y$. Then on $W$ we have $g=\varphi-t+p$ for some pluriharmonic function $p$ on $W$. Since $t$ and $p$ are $C^{\infty}, g$ is locally summable as well as $\varphi$. Now for any function $h$ on $Y$ we write $\tilde{h}=h f$. Then with $W$ as above we have $\tilde{t}=\tilde{\varphi}-\tilde{g}+\tilde{p}$ on $\tilde{W}=f^{-1}(W)$, where all the functions involved are locally summable since $\tilde{t}$ and $\tilde{p}$ are $C^{\infty}$ and $\tilde{\varphi}$ is plurisubharmonic by our assumption that $f(X) \nsubseteq S_{\propto s}(\tau)$. So we can apply $d d^{c}$ on both sides of the equality considering each term as currents. Then we obtain $f^{*} \tau^{\prime}=d d^{c} \tilde{t}=d d^{c} \tilde{\varphi}-d d^{c} \tilde{g}+d d^{c} \tilde{p}=d d c^{c} \tilde{\varphi}-d d^{c} \tilde{g}$ on $\tilde{W}$. Since $\tilde{g}$ is a global function on $X$, this shows that $f^{*} \beta$ is represented by $f^{*} \tau$ by the definition of $f^{*} \tau$.

Q.E.D.

Corollary 2.3. The set of positive classes is preserved under a bimeromorphic map, i.e., if $f: X \rightarrow Y$ is a bimeromorphic map of complex manifolds, then $f_{*}\left(P_{0}(X)\right) \subseteq P_{0}(Y)$ and $f^{*}\left(P_{0}(Y)\right) \subseteq P_{0}(X)$, and similarly for $P(X)$ and $P(Y)$.

Before proceeding we note the following consequence of Lemma 2.2. Let $C$ be a compact irreducible curve on a complex manifold $X$, and $j: C \rightarrow X$ the inclusion. Then we have the natural isomorphism $H^{2}(C, \mathbb{R}) \cong \mathbb{R}$ induced by the complex structure of $C$. Let $\sigma$ be a positive current on $X$ such that $C \not$ $S_{\diamond}(\sigma)$. Let $\sigma^{\prime} \in H^{2}(X, \mathbb{R})$ be defined by $\sigma$ and $\bar{\sigma}=j^{*} \sigma^{\prime} \in H^{2}(C, \mathbb{R})$. Then $\bar{\sigma} \geqq 0$ with respect to the above isomorphism. In fact, laking the normalization $\widetilde{C}$ of $C$ and observing the natural isomorphism $H^{2}(C, \mathbb{P}) \cong H^{2}(\tilde{C}, \mathbb{R})$ we get this easily from Lemma 2.2.

Let $E$ be a complex space and $S$ a subset of $E$. Then $S$ is called thin if for each point $v \in E$ there is a neighborhood $U$ of $v$ in $E$ and a nowhere dense analytic subset $A$ of $U$ such that $S \cap U \subseteq A$.

Lemma 2.4. Let $f: X \rightarrow Y$ be a bimeromorphic morphism of complex manifolds. Let $E$ be the exceptional set of $f$ and $E_{v}, v=1,2, \ldots$, be the $i r$ reducible components of $E$. Let $\sigma \in \widetilde{P}(X)$ such that $S(\sigma) \cap E$ is a thin subset 
of $E$. Then there is a unique sequence $\left(r_{v}\right)$ of nonnegative real numbers $r_{v}$ such that

$$
f^{*} f_{*} \sigma-\sigma=\sum_{v} r_{v}\left\langle E_{v}\right\rangle
$$

where $\left\langle E_{v}\right\rangle$ denotes the integration current on $E_{v}$. Moreover $r_{v} \neq 0$ if the following condition is satisfied: There is a compact irreducible curve $C$ in $E_{v}$ such that $C \leqq f^{-1}(y)$ for some $y \in Y, C \nsubseteq E_{\mu}$ for any $\mu \neq v, C \nsubseteq S(\sigma)$ and that the natural image of $\sigma$ in $H^{2}(C, \boldsymbol{R})$ is nonzero.

For the proof we need two lemmas.

Lemma 2.5. Let $X$ be a complex manifold and $E$ an analytic subset of $X$ of pure codimension 1 . Let $E_{v}, v=1,2, \ldots$, be the irreducible components of $E$. Let $\sigma$ be a real closed locally flat $(1,1)$-current on $X(c f .[8,2.1])$ whose support is contained in $E$. Then there exists a unique real number $r_{v}$ for each $v$ such that

$$
\sigma=\sum_{v} r_{v}\left\langle E_{v}\right\rangle
$$

Proof. See [8, Prop. 3.1.3].

Lemma 2.6. Let $X$ be a complex manifold and $\varphi_{1}, \varphi_{2}$ plurisubharmonic functions on $X$. Let $E$ be an analytic subset of $X$ with $E \neq X$. Suppose that $\varphi_{1}-\varphi_{2}$ is plurisubharmonic on $X-E$ and $S\left(\varphi_{2}\right) \cap E$ is a thin subset of $E$. Then $\varphi_{1}-\varphi_{2}$ is plurisubharmonic on the whole $X$.

Proof. Let $U=X-S\left(\varphi_{2}\right) \cap E$. Since $\varphi_{1}$ is upper semicontinuous and $\varphi_{2}$ is locally bounded from below on $U, \varphi_{1}-\varphi_{2}$ is locally bounded from above on $U$. Let $\psi=\varphi_{1}-\left.\varphi_{2}\right|_{U-E}$. Then $\psi$ is plurisubharmonic on $U-E$ and bounded from above on any compact subset of $U$. Hence by a theorem of Grauert and Remmert (cf. [10, p. 35, Theorem 4] or [14]), $\psi$ extends to a unique plurisubharmonic function $\tilde{\psi}$ on $U$. Then since $\tilde{\psi}+\varphi_{1}=\varphi_{2}$ on $U-E$ and both terms are plurisubharmonic on $U$, they coincide on $U$. Thus $\varphi_{1}-\varphi_{2}=\tilde{\psi}$ and is plurisubharmonic on $U$. Then since $S\left(\varphi_{2}\right) \cap E$ is locally contained in an analytic subset of codimension $\geqq 2$ in $X$, by another theorem of Grauert and Remmert $\left[14\right.$, p. 337] together with the argument as above we get that $\varphi_{1}-\varphi_{2}$ is plurisubharmonic on the whole $X$.

Q.E.D.

Proof of Lemma 2.4. Since the problem is local, we may assume that $f_{*} \sigma=d d^{c} \varphi$ for some plurisubharmonic function $\varphi$ on $Y$. Let $\tilde{\varphi}=\varphi \cdot f$. Then $f^{*} f_{*} \sigma$ is the current defined by $d d^{c} \tilde{\varphi}$ on $X$. Let $x \in X$ be an arbitrary point. 
Take a neighborhood $V$ of $x$ and a plurisubharmonic function $\varphi^{\prime}$ on $V$ such that $\left.\sigma\right|_{V}=d d^{c} \varphi^{\prime}$. Then $\tilde{\varphi}-\varphi^{\prime}$ is pluriharmonic on $V-E \cap V$. Since $S\left(\varphi^{\prime}\right) \cap E$ is thin in $E$ by assumption, we conclude by Lemma 2.6 that $\tilde{\varphi}-\varphi^{\prime}$ is plurisubharmonic on the whole $V$. Then by Lemma 2.5 there is a unique nonnegative real number $r_{v}$ for each $v$ such that $\left.\left(d d^{c} \tilde{\varphi}-\sigma\right)\right|_{v}=d d^{c} \tilde{\varphi}-d d^{c} \varphi^{\prime}=\sum_{v} r_{v}\left\langle E_{v} \cap V\right\rangle$ as currents on $V$. In fact, since a positive current is representable by integration [10, IV, Prop. 7], the $d$-closed positive current $d d^{c} \tilde{\varphi}-d d^{c} \varphi^{\prime}$ is locally normal and hence is locally flat on $V$ (cf. $[8,2.1]$ ). Since $x$ was arbitrary, this shows the desired equality.

Now suppose that there is a compact irreducible curve $C$ in $E_{v}$ satisfying the condition of the lemma. Let $\sigma^{\prime} \in H^{2}(X, \mathbb{R})$ be the class defined by $\sigma$ and $\bar{\sigma}=j^{*} \sigma^{\prime} \in H^{2}(C, \mathbb{R})$ where $j: C \rightarrow X$ is the inclusion. Let $\bar{e}_{v} \in H^{2}(C, \mathbb{R})$ be the real first chern class of $\left.\left[E_{v}\right]\right|_{c}$. Via the natural isomorphism $H^{2}(C, \mathbb{R}) \cong \mathbb{R}$ regard $\bar{\sigma}$ and $\bar{e}_{v}$ as real numbers. Then $\bar{\sigma}>0$ by our assumption that $C \nsubseteq S(\sigma)$ (and hence the natural image of $\sigma$ in $H^{2}(C, \boldsymbol{R})$ is nonzero) and by the remark after Corollary 2.3. Further if $\nu \neq \mu$, then $\bar{e}_{v} \geqq 0$ since $C \nsubseteq E_{v}$. Thus $r_{\mu} \bar{e}_{\mu}$ $=-\bar{\sigma}-\sum_{v \neq \mu} r_{v} \bar{e}_{v}<0$. Hence $r_{\mu} \neq 0$.

Q.E.D.

Remark. ${ }^{1)}$ The existence of $r_{v}$ with $d d^{c} \tilde{\varphi}-d d^{c} \varphi^{\prime}=\sum_{v} r_{v}\left\langle E_{v} \cap V\right\rangle$ as above can also be proved more elementarily as follows. Take a smooth point $x \in E_{v}-\cup_{\mu \neq \nu} E_{\mu}$ and a polydisc neighborhood $x \in U$ with local coordinates $z_{1}, \ldots, z_{n}$ around $x$ satisfying $\left\{z_{1}=0\right\}=U \cap E_{v}$. Let $S=\left\{\left|z_{1}\right|=\varepsilon, z_{2}=\cdots=z_{n}=0\right\}$ for a sufficiently small $\varepsilon>0$. Let $r_{v}=\int_{S} d\left(\tilde{\varphi}-\varphi^{\prime}\right)$. Then $\tilde{\varphi}-\varphi^{\prime}-\left(r_{v} / 2 \pi\right) \log \left|z_{1}\right|$ is pluriharmonic on $U$. Then $\pm\left(d d^{c} \tilde{\varphi}-d d^{c} \varphi^{\prime}-\sum_{v} r_{v}\left\langle E_{v} \cap V\right\rangle\right.$ are both semipositive as in the proof of Lemma 2.6. From this we get the desired equality.

\section{§3. Main Theorem and Corollaries}

Let $X$ be an irreducible complex space and $F$ an analytic subset of $X$ of codimension $\geqq 2$. Let $\mathscr{I}$ be a coherent sheaf of ideals of $\mathcal{O}_{X}$ with support coinciding with $F$, i.e., $\mathscr{I}_{x}=\mathcal{O}_{X, x}$ if and only if $x \notin F$. Let $h: Z \rightarrow X$ be the monoidal transformation with center $\mathscr{I}$. Let $E=h^{-1}(F)$ with reduced structure, $E_{v}$, $v=1,2, \ldots$, be the irreducible components of $E$ and $\mathscr{I}_{v}$ the ideal sheaves of $E_{v}$. Suppose that $Z$ is nonsingular. Then $\mathscr{I}_{v}$ are locally principal and $\mathscr{J}:=h^{-1}(\mathscr{I})$

1) This is pointed out by the refee of this paper. 
is written in the form $\mathscr{J}=\mathscr{I}_{1}^{a_{1}} \mathscr{I}_{2}^{a_{2}} \cdots$ for some positive integers $a_{v}$. Note that $\mathscr{J}$ is $h$-very ample.

Lemma 3.1. The notation being as above, let $\left(r_{1}, r_{2}, \ldots\right)$ be a sequence of non-negative real numbers with at least one $r_{v} \neq 0$, say $r_{1} \neq 0$. Then for any open subset $U$ of $X$ such that $h\left(E_{1}\right) \cap U \neq \emptyset$, there is a compact irreducible curve $B$ on $Z$ contained in $h^{-1}(u)$ for some $u \in U \cap F$ such that $\sum_{v} r_{v} e_{v, B}<0$, where $e_{v, B}=c_{1}\left(\left.\left[E_{v}\right]\right|_{B}\right) \in H^{2}(B, \boldsymbol{R})$ naturally identified with $\boldsymbol{R}$.

Proof. Let $\tilde{U}=h^{-1}(U)$. Let $\mathfrak{N}=\left\{v ; E_{v} \cap \tilde{U} \neq \varnothing\right\}$. We may assume that $U$ is relatively compact so that $\mathfrak{N}$ is finite. Take $\mu \in \mathfrak{N}$ such that $r_{\mu} / a_{\mu}$ is maximal in the set $\left\{r_{v} / a_{v} ; v \in \mathfrak{N}\right\}$. By our assumption $r_{\mu}>0$. Take any compact irreducible curve $B$ in $E_{\mu}$ such that it is contained in $f^{-1}(u)$ for some $u \in U \cap F$ and $B \nsubseteq E_{v}$ for any $v \neq \mu$. Then it is clear that $e_{v, B} \geqq 0$ for every $v \neq \mu$. Hence $\sum_{v} r_{v} e_{v, B}=\sum_{v}\left(r_{v} / a_{v}\right) a_{v} e_{v, B} \leqq \sum_{v}\left(r_{\mu} / a_{\mu}\right) a_{v} e_{v, B}=r_{\mu} / a_{\mu} \sum a_{v} e_{v, B}$, while the last term $<0$ since $\mathscr{J}$ is $h$-very ample and $\mathscr{J} \cong \mathcal{O}_{X}\left(\prod_{v}\left[E_{v}\right]^{-a_{v}}\right)$.

Q.E.D.

Let $X$ be a complex manifold. Then a real $C^{\infty}(1,1)$-form $\phi$ on $X$ is called positive (resp. semipositive) at $x \in X$ if for any local coordinates $\left(z_{1}, \ldots, z_{n}\right)$ around $x, \phi$ has the form $\phi=\sqrt{-1} \sum \phi_{\mu \bar{v}} d z_{\mu} \wedge d \bar{z}_{v}$ with $\left(\phi_{\mu \bar{v}}\right)$ a positive definite (resp. semipositive) Hermitian matrix at $x$ where $n=\operatorname{dim} X . \phi$ is called positive (resp. semipositive) on $X$ if it is so at each point of $X$. Let $\alpha \in H^{1,1}(X)$ (resp. $H^{2}(X, \boldsymbol{R})$ ). Then $\alpha$ is called positive (resp. semipositive) if $\alpha$ is represented by a positive (resp. semipositive) real closed $C^{\infty}$ form of type $(1,1)$. A positive class in $H^{2}(X, \boldsymbol{R})$ also is called a Kähler class. Note that if $\alpha$ is semipositive, then $\alpha$ is current-positive with $S(\alpha)=\varnothing$. Further we recall that usually a line bundle on $X$ is called (semi) positive if its refined chern class $\hat{c}(L)$ is so.

Let $f: X \rightarrow Y$ be a bimeromorphic map of complex manifolds and $E^{(i)}$ the exceptional set of $\pi_{i}, i=1,2$ where $\pi_{1}: \Gamma \rightarrow X$ (resp. $\pi_{2}: \Gamma \rightarrow Y$ ) is the natural projection of the graph $\Gamma$ of $f$ onto $X$ (resp. $Y$ ). Then we call a common irreducible component of $E^{(1)}$ and $E^{(2)}$ a biexceptional component of $f$. Further we set $E(f)=\cup \pi_{1}\left(E_{i}^{(2)}\right), F(f)=\pi_{1}\left(E^{(1)}\right)$ and $S(f)=E(f) \cup F(f)$, where in the first equality the union is taken over all the irreducible components $E_{i}^{(2)}$ of $E^{(2)}$ which are not biexceptional components of $f$. Thus $E(f)$ is of pure codimension 1 in $X$ and $\operatorname{codim} F(f) \geqq 2$ in $X[6]$. We call $E(f), F(f)$ and $S(f)$ the exceptional set, the set of indeterminacy, and the singular set, respectively, of $f$. Clearly $f$ is holomorphic (resp. locally isomorphic) at $x \in X$ if and only if $x \notin F(f)$ (resp. $S(f)$ ). 
Theorem 3.2. Let $f: X \rightarrow Y$ be a bimeromorphic map of complex manifolds. Let $F_{k}$ be an irreducible component of $F(f)$ with $F_{k} \equiv E(f)$. Suppose that there exists a semipositive class $\alpha \in H^{2}(X, \mathbb{R})$ with the following property; 1) $\alpha$ admits $a C^{\infty}$ representative which is semipositive everywhere and positive at some point $x \in F_{k}$, and 2) $S\left(f_{*} \alpha\right) \cap S\left(f^{-1}\right)=\emptyset$ where $S\left(f_{*} \alpha\right)$ is the set of base points of $f_{*} \alpha \in P(Y)$. Then there is no biexceptional component $E_{0}$ of $f$ such that $\pi_{1}\left(E_{0}\right)=F_{k}$.

Proof. By [7] together with resolution we can find a complex manifold $Z$ with bimeromorphic morphisms $h_{1}: Z \rightarrow X$ and $h_{2}: Z \rightarrow Y$ such that $f=h_{2} h_{1}^{-1}$ and $h_{1}$ is locally a monoidal transformation with center a cohcrent analytic sheaf of ideals of $\mathcal{O}_{X}$ with support contained in $F(f)$. Let $E^{(i)}$ be the exceptional sets of $h_{i}$ and $E_{v}^{(i)}, v=1,2, \ldots$, be the irreducible components of $E^{(i)}$. Since $h_{1}^{*} \alpha$ is a semipositive class in $H^{2}(Z, \mathbb{R}$ ), applying Lemma 2.4 to (any positive currents representing) $h_{1}^{*} \alpha$ and to $h_{2}: Z \rightarrow Y$ we get in $H^{2}(Z, \mathbb{R})$ the relation

$$
h_{2}^{*} h_{2 *} h_{1}^{*} \alpha-h_{1}^{*} \alpha=\sum_{v} r_{v} e_{v}^{(2)}
$$

for some nonnegative real numbers $r_{v}$, where $e_{v}^{(2)}=c_{1}\left(\left[E_{v}^{(2)}\right]\right) \in H^{2}(Z, \mathbb{R})$ is the real first chern class of the line bundle $\left[E_{v}^{(2)}\right]$. Now let $g: Z \rightarrow \Gamma$ be the natural map of $Z$ onto the graph $\Gamma$. First we show that $r_{v} \neq 0$ for any $v$ such that $h_{1}\left(E_{v}^{(2)}\right)$ $=F_{k}$ and $g\left(E_{v}^{(2)}\right)$ is a biexceptional component of $f$. In fact for any such $v$ let $z \in E_{v}^{(2)}$ be any point with $h_{1}(z)=x$. Replacing $x$ by a point of $F_{k}$ in a small neighborhood of $x$ if necessary we may assume that $z \notin E_{\mu}^{(2)}$ for any $\mu \neq v$. Since $N:=h_{2}^{-1}\left(h_{2}(z)\right)$ is a compact Moishezon space, and $g\left(E_{v}^{(2)}\right)$ is a biexceptional component of $f$, there is a compact irreducible curve $C$ in $N$ passing through $z$ and not contained in $h_{1}^{-1}(x)$. (Use the fact that there is a bimeromorphic morphism $u: \tilde{N} \rightarrow N$ with $\tilde{N}$ projective.) Let $C^{\prime}=h_{1}(C)$. By our choice of $C, C^{\prime}$ is a compact irreducible curve in $F_{k}$ passing through $x$. Since $x \in C^{\prime}$, the image $\bar{\alpha}$ of $\alpha$ in $H^{2}\left(C^{\prime}, \boldsymbol{R}\right)$ is nonzero by the condition 1). Since the induced $\operatorname{map} \bar{h}_{1}^{*}: H^{2}\left(C^{\prime}, \boldsymbol{R}\right) \rightarrow H^{2}(C, \boldsymbol{R})$ is clearly injective, we get that $j^{*} h_{1}^{*}(\alpha)=\bar{h}_{1}^{*}(\bar{\alpha}) \neq 0$ in $H^{2}(C, \mathbb{R})$ where $j: C \rightarrow Z$ is the inclusion. Thus by Lemma $2.4 r_{v} \neq 0$ as was desired.

Therefore the theorem will be proved if we show that $r_{v}=0$ for any $E_{v}^{(2)}$ with $h_{1}\left(E_{v}^{(2)}\right)=F_{k}$. Let $x^{\prime}$ be any point of $F_{k}$ with $x^{\prime} \notin F_{j}$ for any $j \neq k$, where $F_{j}, j=1,2, \ldots$, are the irreducible components of $F(f)$. Take any relatively compact neighborhood $U$ of $x^{\prime}$ in $X$ such that 


$$
U \cap E(f)=\varnothing, \quad \text { and } \quad U \cap F_{j}=\varnothing, \quad j \neq k
$$

and that $h_{1 \mid \tilde{U}}: \tilde{U} \rightarrow U, \tilde{U}=h_{1}^{-1}(U)$, is the monoidal transformation of $U$ with center a coherent sheaf of ideals $\mathscr{I}$ of $\mathcal{O}_{U}$ with support contained in $U \cap F_{k}$. (Note that $F_{k} \nsubseteq E(f)$.) Let $A_{1}, \ldots, A_{m}$ be the irreducible components of $h_{1}^{-1}\left(F_{k} \cap U\right)$ with reduced structure. Then there is a natural bijective correspondence between the set $\left\{A_{s}\right\}_{1 \leqq s \leqq m}$ and the set of irreducible components $E_{\mu i}^{(1)}$ of $E_{\mu}^{(1)} \cap \tilde{U}, \mu=1,2, \ldots$ In particular for each $s$ we have unique $\mu=\mu(s)$, $i=i(s)$ such that $A_{s}=E_{\mu i}^{(1)} \cap \tilde{U}$. Then define a real number $r_{s}$ by $r_{v}$ if $E_{\mu}^{(1)}=E_{v}^{(2)}$ for some $E_{v}^{(2)}$ (which is unique if any), and $r_{s}=0$, otherwise. Suppose that $r_{s} \neq 0$ for some $s$. Then by Lemma 3.1 there is a compact irreducible curve $B$ on $\tilde{U}$ such that

$$
B \cong h_{1}^{-1}(u) \text { for some } u \in U \cap F_{k}
$$

and that if $\alpha_{s}$ are the real first chern classes of the line bundles $\left[A_{s}\right]$ in $H^{2}(\tilde{U}, \boldsymbol{R})$ and $\bar{\alpha}_{s}$ the natural images of them in $H^{2}(B, \boldsymbol{R})$, then we have $\sum_{s} r_{s} \bar{\alpha}_{s}<0$ with respect to the natural isomorphism $H^{2}(B, \boldsymbol{R}) \cong \boldsymbol{R}$. From our definition of $r_{s}$ this is easily seen to be equivalent to

$$
\sum_{v \in \mathfrak{R}} r_{v} \bar{e}_{v}^{(2)}<0
$$

where $\mathfrak{N}=\left\{v ; h_{1}\left(E_{v}^{(2)}\right)=F_{k}\right\}$ and $\bar{e}_{v}^{(2)}$ denotes the natural image of $e_{v}^{(2)}$ in $H^{2}(B, \boldsymbol{R})$. On the other hand, in view of (10) and (11), from (9) we have $\sum_{v} r_{v} \bar{e}_{v}^{(2)}=c^{*} h_{2}^{*} h_{2 *} h_{1}^{*} \alpha \geqq 0$, for $h_{2}^{*} h_{2 *} h_{1}^{*} \alpha$ is current-positive by 1) and Corollary 2.3, and $S\left(h_{2}^{*} h_{2 *} h_{1}^{*} \alpha\right) \cap B=\varnothing$ by 2) and Lemma 2.2, where $6: B \rightarrow Z$ is the natural inclusion. This contradicts (12). Hence $r_{s}=0$ for all $1 \leqq s \leqq m$. From the definition of $r_{s}$ we then get our assertion, and hence the theorem is proved.

Remark. As follows from the above proof the conditions 1) and 2) of the theorem can be weakened to the following 1$)^{\prime}$ and 2$)^{\prime}$ respectively. 1$)^{\prime} \alpha$ is current-positive (but may not be semipositive) with $\iota^{*} \alpha \geqq 0$ for any compact irreducible curve $B$ contained in $F_{k}$ with respect to the natural isomorphism $H^{2}(B, \boldsymbol{R}) \cong \boldsymbol{R}$, where $\iota^{*}: H^{2}(X, \boldsymbol{R}) \rightarrow H^{2}(B, \boldsymbol{R})$ is the natural map induced by the inclusion $c: B \rightarrow X$ and 2$)^{\prime} S\left(f_{*} \alpha\right) \cap S\left(f^{-1}\right)$ is discrete.

Let $f: X \rightarrow Y$ be a bimeromorphic map of complex manifolds and $g=f^{-1}$. Then we say that $f$ is semi-holomorphic if $E(g)=\emptyset$, or equivalently, codim $S(g)$ $\geqq 2$. Clearly a semi-holomorphic bimeromorphic map $f$ with no biexceptional component is holomorphic. Further if $f$ is holomorphic and $g$ is semi-holomorphic, then $f$ is biholomorphic. 
We say that a line bundle $L$ on a complex manifold $X$ is base point free if the common zero of all the holomorphic sections of $L$ is empty. If $L$ is base point free and $X$ is compact, then $L$ is semipositive in the sense mentioned above.

Corollary 3.3. Let $f: X \rightarrow Y$ be a bimeromorphic map of complex manifolds and $g=f^{-1}$. Suppose that $g$ is semi-holomorphic. Then $g$ is actually holomorphic if either of the following conditions is satisfied. 1) $X$ is a Kähler manifold with a Kähler class $\alpha \in H^{2}(X, \mathbb{R})$ such that $f_{*} \alpha$ is semipositive. 2) $X$ is a projective manifold with an ample line bundle $L$ such that $\left(f_{*} L\right)^{\otimes m}$ is base point free for some $m>0$.

For a complex manifold $Z$ we denote by $K_{Z}$ the canonical bundle of $Z$ and $c_{1}(Z)$ is the real first chern class of $Z$.

Lemma 3.4. Let $f: X \rightarrow Y$ be a bimeromorphic map of complex manifolds. Suppose that the linear system $\left|m K_{Y}\right|$ has no fixed component for some $m>0$, i.e., the common zero of elements of $H^{0}\left(Y, K_{Y}^{\otimes m}\right)$ has no component of codimension 1. Then $f$ is semi-holomorphic.

Proof. In general let $h: Z \rightarrow Z^{\prime}$ be a bimeromorphic map of complex manifolds. Then for any integer $m>0$ we have a natural isomorphism $h^{*}$ : $H^{0}\left(Z^{\prime}, K_{Z}^{\otimes^{\prime}}\right) \rightarrow H^{0}\left(Z, K_{Z}^{\otimes^{m}}\right)$. In fact, if $U=Z-F(h)$, then for any $\varphi \in H^{0}\left(Z^{\prime}\right.$, $\left.K_{Z}^{\otimes m}\right), h^{*} \varphi$ is the unique extension of $\left(\left.h\right|_{U}\right)^{*} \varphi$ to $Z$ (cf. [9, VIII §2]). Then $\left(h^{-1}\right)^{*}$ gives the inverse to $h^{*}$. Now take any $\omega \in H^{0}\left(Y, K_{Y}^{\otimes m}\right)$. Then letting $g=f^{-1}$ we have $\omega=g^{*} f^{*} \omega$. Hence $\left.\omega\right|_{Y-F(g)}$ vanishes on $E(g) \cap(Y-F(g))$, and therefore if $E(g) \neq \varnothing$, then $E(g)$ is contained in the common zero of the elements of $H^{0}\left(Y, K_{Y}^{\otimes_{m}}\right)$, contradicting our assumption. Thus $E(g)=\varnothing$.

Q.E.D.

Proposition 3.5. Let $X$ be a projective manifold with $c_{1}(X)<0$ and $Y$ a Moishezon manifold which is bimeromorphic to $X$. Then if $c_{1}(Y) \leqq 0$ and $Y \varsubsetneqq X$, then $\left|m K_{Y}\right|$ have fixed components for all $m>0$.

Proof. Let $f: X \rightarrow Y$ be any bimeromorphic map and $g=f^{-1}$. Suppose that $\left|m K_{Y}\right|$ has no fixed component for some $m>0$. Then by Lemma $3.4 f$ is semi-holomorphic. Hence we have an isomorphism $f: X-E(f) \cong Y-F(g)$, so $\left.f_{*} c_{1}(X)\right|_{Y-F(g)}=c_{1}(Y-F(g))=\left.c_{1}(Y)\right|_{Y-F(g)}$. Then, since $F(g)$ is of codimension $\geqq 2$ in $Y$ so that the natural restriction $H^{2}(Y, \boldsymbol{R}) \rightarrow H^{2}(Y-F(g), \mathbb{R})$ is isomorphic, we have $f_{*} c_{1}(X)=c_{1}(Y)$. On the other hand, since the high multiple of $K_{X}$ is very ample, again by Lemma $3.4 \mathrm{~g}$ is semi-holomorphic. Hence by 
Corollary $3.3 \mathrm{~g}$ is holomorphic, and hence isomorphic since $f$ is semi-holomorphic. Thus $X \cong Y$.

Q.E.D.

In particular for any compact complex manifold $Z$ which is bimeromorphic to $X$ and $₹ X,\left|m K_{Z}\right|$ have base points for all $m>0$, which also follows directly from the arguments of Peters in [13]. On the other hand, the author knows no example of a Moishezon manifold with $c_{1}(Z) \leqq 0$ such that $\left|m K_{Z}\right|$ have fixed components for all $m>0$.

For a compact complex manifold $Z$ we set $h^{1,1}(Z)=\operatorname{dim}_{C} H^{1,1}(Z)$ and $\rho(Z)=\operatorname{dim}_{\boldsymbol{C}}\left(H^{2}(Z, Q) \cap \lambda\left(H^{1,1}(Z)\right)\right.$ ) (the Picard number of $Z$ ). Recall that $\lambda$ is injective when $Z$ is bimeromorphic to a compact Kähler manifold.

Proposition 3.6. Let $X$ be a compact Kähler (resp. projective) manifold with $h^{1,1}(X)=1$ (resp. $\rho(X)=1$ ) and with $c_{1}(X)=0$. Let $Y$ be any compact complex manifold bimeromorphic to $X$ with $h^{1.1}(Y)=1$ (resp. $\left.\rho(Y)=1\right)$ and with a semipositive class $\beta$ in $H^{2}(Y, \boldsymbol{R})$ (resp. $H^{2}(Y, \boldsymbol{Q})$ ). Then every bimeromorphic map $f: X \rightarrow Y$ is isomorphic.

Proof. Let $f: X \rightarrow Y$ be any bimeromorphic map and $g=f^{-1}$. Then by [1, Theorem 3'] (or directly from [3, Prop. 6.6]) $K_{X}^{\otimes m}$ is trivial for some $m>0$ so that by Lemma $3.4 \mathrm{~g}$ is semi-holomorphic. Since $h^{1,1}(X)=h^{1,1}(Y)$ (resp. $\rho(X)=\rho(Y)$ ), by considering $Z$ as in the proof of Theorem 3.2, from Proposition 1.1 we infer readily that $f$ also is semi-holomorphic. On the other hand, let $\alpha$ be a Kähler class in $H^{2}(X, \mathbb{R})$ (resp. $H^{2}(X, \boldsymbol{Q})$ ). Then we may write $f_{*} \alpha=r \beta$ for some $r \in \mathbb{R}$ (resp. $Q$ ). Then since $\lambda$ is injective on $Y$, by Lemma 2.1 and Corollary $2.3 r \geqq 0$. Hence $g$ is holomorphic by Corollary 3.3. Hence $f$ is biholomorphic.

Q.E.D.

In [2] it was conjectured that every bimeromorphic automorphism of a projective manifold $X$ with trivial canonical bundle is necessarily isomorphic. The above result gives a partial answer to this conjecture (cf. also [2, Theorem 5]).

\section{§4. Kählerian Analogue of a Theorem of Matsusaka-Mumford}

Besides Lemma 3.4 there is a case in which a given bimeromorphic map is necessarily semi-holomorphic. First we recall a definition. Let $Z$ be a compact complex manifold and $\pi: V \rightarrow Z$ a holomorphic vector bundle over $Z$ of finite 
rank $>1$. Then we denote by $\mathbb{P}(V)$ the associated projective bundle over $Z$. Then a compact irreducible complex space $B$ is called ruled if it is bimeromorphic to $\boldsymbol{P}(V)$ for some $Z$ and $V$ as above.

Lemma 4.1. Let $f: X \rightarrow Y$ be a bimeromorphic map of complex manifolds. Suppose that $E(f)$ is compact. Then every irreducible component of $E(f)$ is ruled.

Proof. Since $E(f)$ is compact, $F\left(f^{-1}\right)$ also is compact. Hence by Hironaka [7] there is a finite succession of monoidal transformations with nonsingular centers $\sigma_{i}: X_{i+1} \rightarrow X_{i}, 1 \leqq i \leqq s, X_{1}=Y$ such that $h=f^{-1} \sigma_{1} \cdots \sigma_{s}: X_{s+1}$ $\rightarrow X$ is a morphism. Let $\tilde{E}$ be the exceptional set of $\sigma=\sigma_{1} \cdots \sigma_{s}$. Then each irreducible component of $\widetilde{E}$ is ruled as well as those of $\sigma_{i}$. Since each irreducible component of $E(f)$ is a bimeromorphic image by $h$ of some irreducible component of $\tilde{E}$ (note that codim $F\left(h^{-1}\right) \geqq 2$ and $E(f)$ is of pure codimension 1 in $X$ ), it is ruled.

Q.E.D.

Lemma 4.2. Let $X$ and $Y$ be complex manifolds, and $A$ and $B$ compact irreducible divisors on $X$ and $Y$ respectively. Let $f: X \rightarrow Y$ be a bimeromorphic map which induces an isomorphism $f^{\prime}: X-A \cong Y-B$. Suppose that $X$ is Kähler with a Kähler class $\alpha \in H^{2}(X, \mathbb{R})$ and that $c_{1}\left(\left.[B]\right|_{B}\right)=0$ in $H^{2}(B, \mathbb{R})$ where $c_{1}$ denotes the first chern class. Suppose further that $f_{*}^{\prime}\left(\left.\alpha\right|_{X-A}\right) \in$ $H^{2}(Y-B, \mathbb{R})$ extends to a semipositive class $\beta$ on $Y$. Then $f$ is isomorphic if either $A$ or $B$ is nonruled.

Proof. Consider the following exact sequence of local cohomology

$$
\longrightarrow H_{B}^{2}(Y, \mathbb{R}) \longrightarrow H^{2}(Y, \mathbb{R}) \stackrel{v}{\longrightarrow} H^{2}(Y-B, \mathbb{R}) \longrightarrow .
$$

Then in view of the natural isomorphism $H^{0}(B, \mathbb{R}) \cong H^{2}(Y, \mathbb{R})$ we infer readily that the kernel of $v$ is generated by $c_{1}([B])$. Now we may replace $Y$ by a tubular neighborhood $U$ of $B$ in $Y$ and $X$ by $f(U)$, so that the natural restriction $H^{2}(Y, \mathbb{R})$ $\rightarrow H^{2}(B, \mathbb{R})$ is isomorphic. Then by assumption $c_{1}([B])=0$. Thus $v$ is injective. Hence the assumption that $f_{*}^{\prime}\left(\left.\alpha\right|_{X-A}\right)=\left.\beta\right|_{Y-B}$ implies that $f_{*} \alpha=\beta$ on $Y$. Hence if $A$ is nonruled, then from Lemma 4.1 and Corollary 3.3 it follows that $g:=f^{-1}$ is holomorphic, and hence is isomorphic, for $g(B)=A$ is of codimension 1 so that $B \nsubseteq E(g)$. So suppose that $B$ is nonruled. Then by Lemma $4.1 \mathrm{~g}$ is locally biholomorphic at a general point $y$ of $B$. Then since $g(Y) \in A$, $A \nsubseteq E(f)$. Hence $E(f)=\varnothing$ and $g$ is semi-holomorphic. Then by the same argument as above the lemma follows. 
A typical application of the above lemma is to the case where $B$ is a fiber of a proper morphism $g: Y \rightarrow S$ of $Y$ onto a disc $S$. In this case, however, the condition can be reduced to a more manageable form under an additional condition on the morphism. So let $X_{i}, i=1,2$, be complex manifolds and $S=\{s \in C ;|s|<1\}$ the unit disc. Let $g_{i}: X_{i} \rightarrow S$ be proper flat morphisms with connected fibers. Let $S^{\prime}=S-\{0\}$ and $X_{i}^{\prime}=g_{i}^{-1}\left(S^{\prime}\right)$. For $s \in S$ we write $X_{i s}=g_{i}^{-1}(s)$ with the natural complex structure. We assume that $g_{i}$ are smooth on $X_{i}^{\prime}$ and $X_{i 0}$ are irreducible for $i=1,2$. Let $f: X_{1} \rightarrow X_{2}$ be a bimeromorphic map over $S$ which induces an isomorphism $f^{\prime}: X_{1}^{\prime} \rightarrow X_{2}^{\prime}$. For $s \in S^{\prime}$ we denote by $f_{s}^{\prime}$ the isomorphism $\left.f\right|_{X_{1 s}}: X_{1 s} \rightarrow X_{2 s}$. Further for a cohomology class $\psi \in H^{2}\left(X_{i}, \boldsymbol{R}\right)$, we shall denote by $\psi_{s}, s \in S$, the natural restriction of $\psi$ to $H^{2}\left(X_{i s}, \boldsymbol{R}\right)$.

Theorem 4.3. The notation and assumption being as above, suppose that there exist a Kähler class $\alpha \in H^{2}\left(X_{1}, \boldsymbol{R}\right)$ and a semipositive class $\beta \in H^{2}\left(X_{2}, \boldsymbol{R}\right)$ such that $f_{*}(\alpha)_{s}=\beta_{s}$ for some (and hence all) $s \in S^{\prime}$. Suppose further that either $g_{2}$ is smooth or $b_{1}\left(X_{2 s}\right)=0$ for $s \in S^{\prime}$, where $b_{1}$ denotes the first Betti number. Then $f$ is isomorphic if either of $X_{i 0}$ is nonruled.

Proof. Considering the Leray spectral sequence for the map $g_{2}^{\prime}: X_{2}^{\prime} \rightarrow S^{\prime}$ and the fact that $H^{i}\left(S^{\prime}, R^{q} g_{2 *}^{\prime} \boldsymbol{R}\right)=0, i \geqq 2, q \geqq 0$, we have the exact sequence

$$
0 \longrightarrow H^{1}\left(S^{\prime}, R^{1} g_{2 *}^{\prime} \boldsymbol{R}\right) \longrightarrow H^{2}\left(X_{2}^{\prime}, \boldsymbol{R}\right) \stackrel{\imath}{\longrightarrow} H^{0}\left(S^{\prime}, \boldsymbol{R}^{2} g_{2 *}^{\prime} \boldsymbol{R}\right) \text {. }
$$

First if $b_{1}\left(X_{2 s}\right)=0, s \in S^{\prime}$, then $R^{1} g_{2 *}^{\prime} \boldsymbol{R}=0$ and hence $\tau$ is injective. Thus the condition $f_{*}(\alpha)_{s}=\beta_{s}$ implies that $\left.f_{*} \alpha\right|_{X_{2}^{\prime}}=\left.\beta\right|_{X_{2}^{\prime}}$. Next, if $g_{2}$ is smooth, then it is clear that the composition $H^{2}\left(X_{2}, \boldsymbol{R}\right) \rightarrow H^{2}\left(X_{2}^{\prime}, \boldsymbol{R}\right) \rightarrow H^{0}\left(X^{\prime}, R^{2} g_{2 *}^{\prime} \boldsymbol{R}\right)$ is injective so that we also have $\left.f_{*} \alpha\right|_{X_{2}^{\prime}}=\left.\beta\right|_{X_{2}^{\prime}}$. Now we put $A=X_{10}$ and $B=X_{20}$. Clearly $[B]$ is trivial so that $c_{1}([B])=0$. Hence the proposition follows from Lemma 4.2.

Remark. The above proof shows that the condition $b_{1}\left(X_{2 s}\right)=0$ may be replaced by the condition that $H^{1}\left(S^{\prime}, R^{1} g_{2 *}^{\prime} \boldsymbol{R}\right)=0$. The latter is equivalent to saying that 1 is not in the eigenvalues of the monodromy transformation on $H^{1}\left(X_{s}, \boldsymbol{R}\right)$, which are known to be roots of unity.

In terms of line bundles (instead of chern classes corresponding to them) we can get a more complete result.

Theorem 4.4. Let $g_{i}: X_{i} \rightarrow S, i=1,2$, and $f: X_{1} \rightarrow X_{2}$ be as in Theorem 4.3. Suppose that there exist line bundles $L_{i}$ on $X_{i}$ such that $c_{1}\left(L_{1}\right)\left(\operatorname{resp} . c_{1}\left(L_{2}\right)\right)$ is 
positive (resp. semipositive), and $L_{1 s} \cong f_{s}^{*} L_{2 s}$ for $s \in S^{\prime}$ where $L_{i s}=\left.L_{i}\right|_{x_{i s}}$. Then $f$ is isomorphic if either $X_{10}$ or $X_{20}$ is nonruled.

Proof. Let $\bar{L}_{1}=f_{*} L_{1}$. Then we show that $\left.c_{1}\left(\bar{L}_{1}\right)\right|_{x_{2}^{\prime}}=\left.c_{1}\left(L_{2}\right)\right|_{x_{2}^{\prime}}$, which would give the proposition as in the proof of the above proposition. We set $L=\bar{L}_{1} \otimes L_{2}^{-1}$ so that $\bar{L}_{1}=L \otimes L_{2}$. Since $\left.L\right|_{X_{2}}$, is trivial, $\left.L\right|_{X_{2}^{\prime}}$ is in the image of the natural map $H^{1}\left(X_{2}^{\prime}, \mathcal{O}_{X_{2}^{\prime}}\right) \cong H^{0}\left(S^{\prime}, R^{1} g_{2 *}^{\prime} \mathcal{O}_{X_{2}^{\prime}}\right) \rightarrow H^{\circ}\left(S^{\prime}, R^{1} g_{2 *}^{\prime} \mathcal{O}_{X_{2}^{\prime}}^{\prime}\right) \cong$ $\left.H^{1}\left(X_{2}^{\prime}, \mathcal{O}_{X_{2}^{\prime}}^{*}\right) \ni L\right|_{X_{2}^{\prime}}$. Thus $c_{1}\left(\left.L\right|_{X_{2}^{\prime}}\right)=0$, so $\left.c_{1}\left(\bar{L}_{1}\right)\right|_{X_{2}^{\prime}}=\left.\left(c_{1}(L)+c_{1}\left(L_{1}\right)\right)\right|_{X_{2}^{\prime}}=$ $\left.c_{1}\left(L_{2}\right)\right|_{X_{2}^{\prime}}$ as was desired.

Q.E.D.

A result of the type considered above was first found by Matsusaka and Mumford [11, Theorem 2] when $g_{i}$ are both smooth and $c_{1}\left(L_{i}\right)$ are both positive, i.e., $L_{i}$ are $g_{i}$-ample.

Proposition 4.5. Let $g_{i}: X_{i} \rightarrow S, i=1,2$, be as in Theorem 4.3. Suppose that $X_{i}$ are Kähler with Kähler classes $\alpha_{i} \in H^{2}\left(X_{i}, \mathbb{R}\right)$ and that the canonical bundles $K_{X_{i}}$ of $X_{i}$ are trivial for $i=1,2$. Let $f: X_{1} \rightarrow X_{2}$ be a bimeromorphic map over $S$ which is isomorphic over $S^{\prime}=S-\{0\}$. Then if $f_{*} \alpha_{1}=\alpha_{2}, f$ is isomorphic.

Proof. Immediate from Lemma 4.3 and Corollary 3.3.

The above proposition is interesting because of the recent result of Persson and Pinkham [12] to the effect that if $g: X \rightarrow S$ is a semi-stable degeneration of compact analytic surfaces with trivial canonical bundles, then there always exists a degeneration $g_{1}: X_{1} \rightarrow S$ bimeromorphic to $g$ such that $K_{X_{1}}$ is trivial.

\section{References}

[1] Bogomolov, F. A., Kähler manifolds with trivial canonical class, Math. USSR Izvestija, 8 (1974), 9-20.

[2] — - On the decomposition of Kähler manifolds with trivial canonical class, Math. USSR Sbornik, 22 (1974), 580-583.

[3] Fujiki, A., On automorphism groups of compact Kähler manifolds, Inventiones math., 44 (1978), 225-258.

[4] _- On the minimal models of complex manifolds, Math. Ann., 253 (1980), $111-128$.

[5] - Coase moduli space for polarized compact Kähler manifolds and polarized algebraic manifolds, to appear.

[6] Grauert, H. and Remmert, R., Zur Theorie der Modifikationen I, Stetige und eigentliche Modifikationen komplexer Räume, Math. Ann., 129 (1955), 274-296.

[7] Hironaka, H., Flattening theorem in complex analytic geometry, Amer. J. Math., 97 (1975), 503-547. 
[ 8] King, J., The currents defined by analytic varieties, Acta Math., 127 (1971), 185220.

[9] Kobayashi, S., Hyperbolic manifolds and holomorphic mappings, Marcel Dekker, 1970.

[10] Lelong, P., Plurisubharmonic functions and positive differential forms, Gordon and Breach, 1969.

[11] Matsusaka, T. and Mumford, D., Two fundamental theorems on deformations of polarized varieties, Amer. J. Math., 86 (1964), 668-684.

[12] Persson, U. and Pinkham, H., Degeneration of surfaces with trivial canonical bundle, Ann. Math., 113 (1981), 45-66.

[13] Peters, K., Über holomorphe und meromorphe Abbildungen gewisser kompakter komplexer Mannigfaltigkeiten, Arch. Math., 15 (1964), 223-231.

[14] Shiffman, B., Extension of positive line bundles and meromorphic maps, Inventions math., 15 (1972), 332-347. 